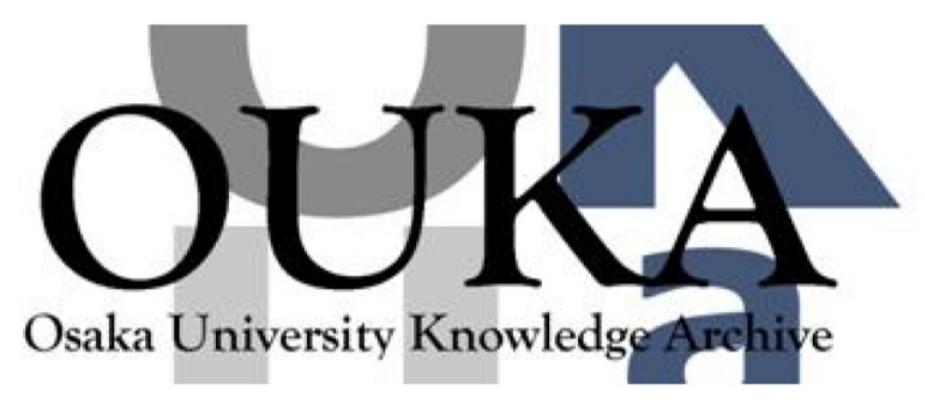

\begin{tabular}{|c|c|}
\hline Title & $\begin{array}{l}\text { Rapid thickness measurements using guided waves } \\
\text { from a scanning laser source }\end{array}$ \\
\hline Author (s) & $\begin{array}{l}\text { Hayashi, Takahiro; Murase, Morimasa; Salim, } \\
\text { Muhammad Nor }\end{array}$ \\
\hline Citation & $\begin{array}{l}\text { The Journal of the Acoustical Society of } \\
\text { America. 126(3) p.1101-p.1106 }\end{array}$ \\
\hline Issue Date & $2009-09$ \\
\hline oaire:version & VoR \\
\hline URL & https://hdl. handle. net/11094/84503 \\
\hline rights & $\begin{array}{l}\text { Copyright } 2009 \text { Acoustical Society of America. } \\
\text { This article may be downloaded for personal use } \\
\text { only. Any other use requires prior permission } \\
\text { of the author and the Acoustical Society of } \\
\text { America. }\end{array}$ \\
\hline Note & \\
\hline
\end{tabular}

Osaka University Knowledge Archive : OUKA

https://ir. Library. osaka-u. ac. jp/

Osaka University 


\title{
Rapid thickness measurements using guided waves from a scanning laser source
}

\author{
Takahiro Hayashi, ${ }^{\text {a) }}$ Morimasa Murase, and Muhammad Nor Salim \\ Faculty of Engineering, Nagoya Institute of Technology, Gokiso, Showa, Nagoya 466-8555, Japan
}

(Received 6 January 2009; revised 13 June 2009; accepted 16 June 2009)

\begin{abstract}
Guided waves have been effectively used for rapid inspections of plates and pipes. However, the guided-wave technique is not generally used for measuring the remaining thickness in a plate and a pipe due to the difficulties in guided-wave motion. Instead, time-consuming and costly direct contact thickness measurements are still used in practice. This study describes a thickness measurement technique using the A0 mode of a Lamb wave generated by a laser source. A finite element analysis of Lamb wave revealed that this mode propagates with small reflections and mode conversions at a rounded shallow defect and has larger amplitude at thinner regions. Using these characteristics, it is experimentally demonstrated that the distributions of plate thickness were obtained from the amplitude of A0 mode generated by a scanning laser source and received by an angle-beam transducer. The resulting distribution images were obtained at extremely high speed compared to the conventional thickness measurements.
\end{abstract}

(C) 2009 Acoustical Society of America. [DOI: 10.1121/1.3177268]

PACS number(s): 43.35.Zc, 43.35.Yb, 43.20.Mv [AJZ]

Pages: 1101-1106

\section{INTRODUCTION}

Low-frequency ultrasonic propagation of guided waves along elongated structures such as plates and pipes has been widely used for nondestructive inspection of inaccessible regions in large structures. ${ }^{1,2}$ Usually, in guided-wave inspection, defects are located by pulse-echo method using the arrival time of defect echoes and the group velocity of the guided waves. In pipe inspection, long-range screening has been realized by the use of the nondispersive axisymmetric torsional mode $T(0,1) .{ }^{3,4}$ Many studies have also examined non-axisymmetric modes for improving pipe inspection. ${ }^{5,6}$ Hayashi and Murase ${ }^{7}$ and Davis and Cawley ${ }^{8}$ further developed defect-imaging techniques by the use of guided waves in a pipe.

However, such fast and cost-effective techniques have not been developed for evaluating remaining thickness of a plate and a pipe, which is the most critical factor in the maintenance of large structures such as tanks and pipeworks. Thus, time-consuming and costly conventional techniques using a contact ultrasonic transducer are still used for thickness measurements.

The present study introduces a rapid thickness measurement technique for plates using guided waves. Many studies in the past discussed the amplitude change in pulse echoes for different defect depths., ${ }^{2,10}$ Generally, the amplitude of an echo signal tends to increase as the ratio of projected area of the defect to its cross-sectional area increases. However, the amplitude is also affected by the width, length, and shape of a defect, ${ }^{11}$ and so in many cases, the amplitude alone cannot be used as an index of the remaining thickness. Such imaging techniques ${ }^{7,8}$ can be used to count defects, but not to evaluate the remaining thickness.

\footnotetext{
a) Author to whom correspondence should be addressed. Electronic mail: hayashi@nitech.ac.jp
}

In this study, the authors use the A0 mode of a Lamb wave generated from a laser source ${ }^{12,13}$ measured by an ultrasonic transducer located remotely from the source, rather than a pulse-echo technique. A laser beam can be emitted far from the inspected area, and the source of the laser-generated ultrasonic wave can be rastered with a galvano scanner. The Lamb wave is detected with angle-beam transducers located far away from the source region. Rapid thickness measurements can thus be carried out.

The paper is organized as follows. First, numerical analyses are carried out to determine the scope of the thickness measurement using a combined model of a semianalytical finite element (SAFE) method ${ }^{6,14-17}$ and a finite element (FE) method. Next, the wave propagation of an A0 mode at a defect in a plate is discussed in detail. Then, images of remaining thickness of a plate are obtained experimentally.

\section{CALCULATION TECHNIQUE FOR GUIDED-WAVE PROPAGATION}

In order to understand guided-wave propagation around defects, a combined SAFE ${ }^{6,14-17}$ and FE model was used in this study. This section briefly describes the combination technique for calculating guide wave propagation and then evaluates the accuracy of the calculation code.

Recently, SAFE has been widely used in guided-wave calculations. ${ }^{6,14-17}$ In that method, only the cross-section of the object is divided into small elements, while the longitudinal direction is expressed in terms of orthogonal functions. Consequently, a smaller number of nodes are required than in the ordinary FE method, so that less memory and shorter calculation times are required. In addition, modal analysis is easier because the wave propagation is described as a sum of guided-wave modes. However, the geometry and material properties must be constant in the longitudinal direction, and 


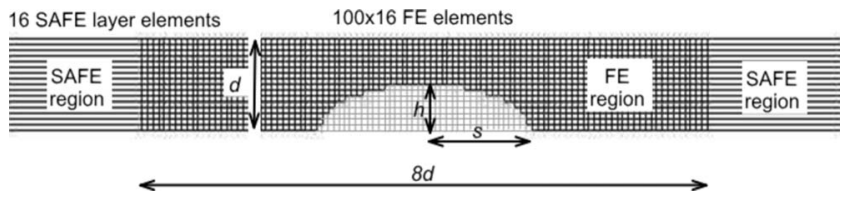

FIG. 1. SAFE and FE meshes used in the combination technique for modeling a plate having a defect.

thus a plate or a pipe with a defect of complex shape cannot be modeled only by the SAFE model. For this reason, a combination technique ${ }^{18-21}$ is here adopted. Various combination techniques have been developed for an effective calculation of Lamb-wave propagation. For example, Al-Nassar et $a l^{18}$ developed the combination technique of an analytical solution of Lamb wave and FE method, Cho and Rose ${ }^{19}$ introduced the combination technique of an analytical solution and boundary element (BE) method, and Galan and $\mathrm{Abascal}^{20}$ used the SAFE and BE models. In this study, the authors adopt the similar combination technique, in which the SAFE is used for intact regions and the ordinary FE method is only used in defect areas.

In order to solve for Lamb-wave propagation in a plate having a defect, such a combination method for a twodimensional elastodynamic problem is used here. As shown in Fig. 1, the defect is described by a FE region divided in both the vertical and horizontal directions into small rectangular elements. At both ends of the FE region, SAFE regions divided into layered elements are connected. In the FE region, a $16 \times 100$ rectangular mesh is used, where the elastic constants of the elements in a dented region are specified to be small. Using a uniform mesh of identical rectangular shapes, the calculations in the FE region require less calculation time and memory. The SAFE and FE regions are connected with continuous displacements and stresses at their boundaries. Displacements and stresses within the plate can be calculated at every frequency step. The frequency domain data are converted to transient wave forms in time domain using inverse fast Fourier transform (FFT).

First of all, in order to evaluate the accuracy of the SAFE and FE combination model, the reflection and transmission factors were calculated and compared with the values obtained by Cho and Rose ${ }^{19}$ and Galan and Abascal ${ }^{20}$ for a steel plate having an elliptical defect of horizontal radius $s$ and vertical radius $h$ for A0 incident mode. These factors can be calculated from the in-plane average power. The displacements and stresses in the SAFE regions determine the inplane average power for the incident, reflected, and transmitted waves. Taking the ratio of the reflected or transmitted waves to the incident wave, the square root of the ratios becomes the reflection and transmission factors, $R$ and $T \cdot{ }^{19,20}$

Figure 2 plots $R$ and $T$ of the A0 and S0 modes for A0 incidence in a steel plate of thickness $d$, longitudinal velocity $c_{L}=5940 \mathrm{~m} / \mathrm{s}$, and transverse velocity $c_{T}=3200 \mathrm{~m} / \mathrm{s}$ with an elliptically rounded dent of $s=1.5 d$ and $h=0.5 d$. The horizontal axis is the product of frequency $f$ and thickness $d$. Cho and Rose ${ }^{19}$ and Galan and Abascal ${ }^{20}$ found the same results for a value of $f d$ below the A1 cutoff frequency of $f d$ $=1.63 \mathrm{MHz} \mathrm{mm}$. This agreement demonstrates that the combination model has sufficient accuracy.

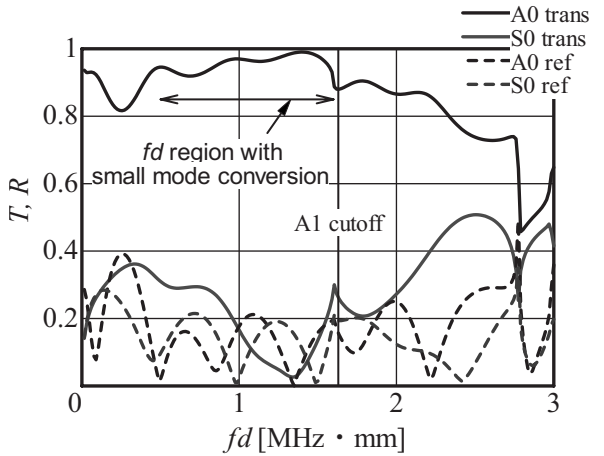

FIG. 2. Transmission and reflection factors for A0 incidence in a steel plate with $c_{L}=5940 \mathrm{~m} / \mathrm{s}$ and $c_{T}=3200 \mathrm{~m} / \mathrm{s}$.

In Fig. 2, below the cutoff frequency, reflections and mode conversions are small, and the incident A0 mode propagates through the defect while keeping its mode shape. This phenomenon is an important characteristic for the thickness measurements described next.

\section{PROPAGATION OF AN AO MODE AT A DENT AND THICKNESS MEASUREMENT}

Displacements and stresses are obtained at every frequency step, and then the reflection and transmission factors $R$ and $T$ are calculated. After collecting displacements at all grid points on the cross-section of a plate, time-domain wave forms are obtained by taking an inverse FFT. Then the wave forms at all grid points can be visualized. ${ }^{17,21}$ In this section, Lamb-wave propagation for A0 incidence is calculated and visualized for explanation of the principles of the thickness measurement technique described in this study.

Wave propagation around an elliptical defect $(s=3 d$ and $h=0.75 d$ ) is calculated for A0 incidence. For the comparison with experimental results shown later, calculations after this section are carried out for an aluminum plate of thickness $d$ $\left(c_{L}=6260 \mathrm{~m} / \mathrm{s}\right.$ and $\left.c_{T}=3080 \mathrm{~m} / \mathrm{s}\right)$. Figure 3 shows the group velocity dispersion curves for the aluminum plate, as well as the frequency spectrum for incident waves ranging below the A1 cutoff $f d$ value where reflections and mode conversions are small.

Wave forms at three different time steps obtained in the calculation are shown in Fig. 4. Gray scale on the surface denotes the displacement in the vertical direction. The incident A0 mode reaches the elliptical defect, and then it propa-

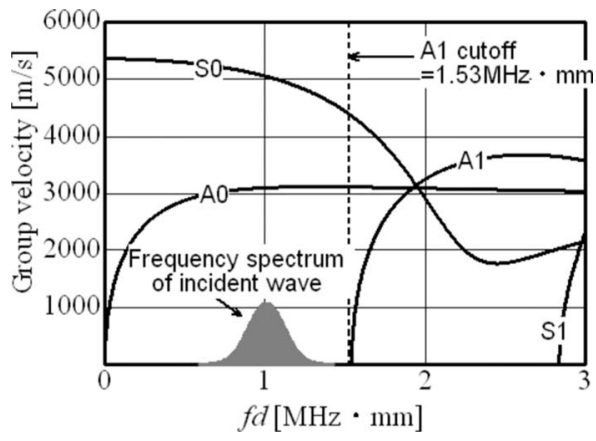

FIG. 3. Group velocity dispersion curves for an aluminum plate, along with the frequency spectrum of the incident wave used in the calculation. 


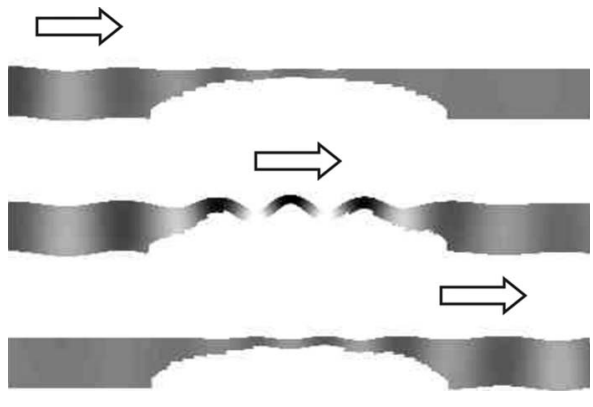

FIG. 4. Propagation of the A0 mode around a defect.

gates with keeping the mode shape of flexural vibrations. When propagating through smaller thickness, the out-ofplane displacement of the A0 mode becomes larger. Finally, the transmitted wave returns to the original amplitude in the intact thick region. This amplitude change is caused by the fact that almost all of the energy of the incident A0 mode propagates through the defect region in this frequency range.

In other words, when an A0 mode in the frequency range of small reflection and mode conversion is incident on a rounded defect, we can roughly estimate the thickness by measuring the normal amplitude at the surface of the defect. Now, assume that the A0 mode emitted from an ultrasonic transducer, such as angle-beam transducers, is detected by a laser interferometer over a surface of an object. Though the A0 mode can be emitted efficiently using the angle-wedge whose angle is determined by Snell's law, laser beam direction and focus must be controlled and adjusted very carefully for stable measurements by the laser interferometer from a distance.

On the other hand, the authors consider the case when normal loading is applied to the surface of a plate and an A0 mode is detected using an angle-beam transducer. Taking into account the reciprocal theorem, the wave form of the A0 mode is identical to the normal vibration detected at the source by the angle-beam transducer. That is, when the normal vibration is applied to a thin region of a plate and an A0 mode in the frequency range of small reflection and mode conversion is detected remotely, the amplitude of the detected wave form becomes large. By contrast, if a vibration is applied at a thick region, the amplitude of the detected signals is small.

When a laser beam is used for ultrasonic generation, it can be easily rastered by mirrors and emitted from a remote distance (e.g., $10 \mathrm{~m}$ away). Therefore, remote rapid thickness measurement can be realized by using a scanning laser source $^{12,13}$ (SLS) and angle-beam transducers to detect an A0 mode in the frequency range of small reflection and mode conversion.

\section{SCOPE OF THE THICKNESS MEASUREMENT}

The thickness measurement technique using a SLS requires an A0 mode propagating through defects with small reflections and mode conversion. Therefore, it can be estimated that this technique has limitations in defect shape,
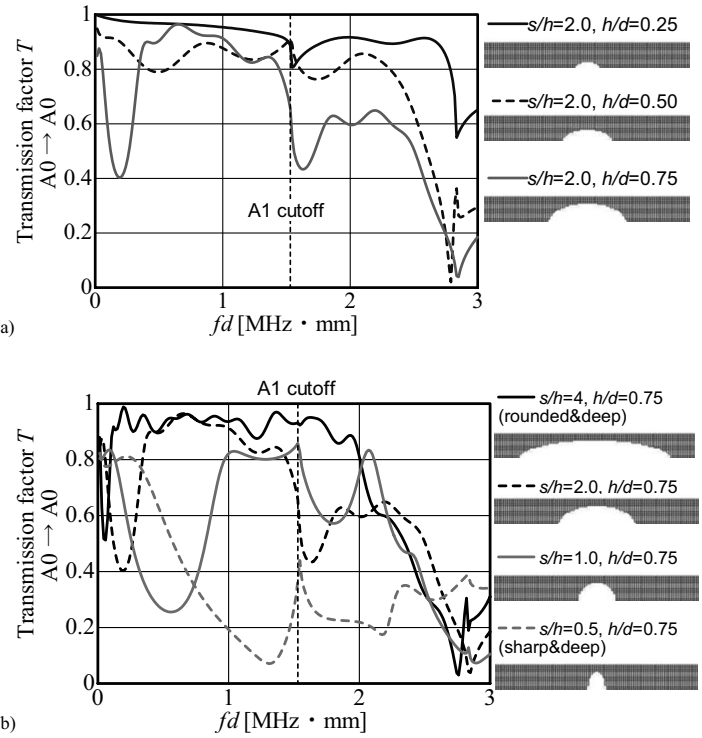

FIG. 5. Transmission factors for A0 incidence at a rounded defect. (a) Different depth but fixed shape. (b) Different shape but fixed depth.

depth, and ultrasonic frequency. In this section, the scope of the measurement is investigated for aluminum plates with defects of various shapes.

First, consider the transmission factors for A0 incidence shown in Fig. 5. Figure 5(a) shows a rounded defect of varying depth for a fixed shape $s / h=2.0$. On the other hand, Fig. 5(b) plots the transmission factors for a deep defect of fixed depth $h / d=0.75$ but variable shape. Above the A1 cutoff, the transmission factors become small as the frequency increases. On the other hand, below the cutoff, the transmission factors are over 0.8 across the $f d$ range of about $0.5-1.4 \mathrm{MHz} \mathrm{mm}$ except sharp and deep defects $s / h=1.0$, $h / d=0.75$ and $s / h=0.5, h / d=0.75$.

Transmission factors for the A0 mode at $f d$ $=1.0 \mathrm{MHz} \mathrm{mm}$ for defects with different $s / h$ and $h / d$ values are plotted in Fig. 6. The general trend is that the transmission factors become smaller for sharper and deeper defects.

Generation of Lamb waves by a SLS and detection of the A0 mode at a left remote intact region are next simulated. When point loading is applied on the surface of a plate, several modes propagate in both directions. Since the amplitude of each mode can be separately calculated in SAFE

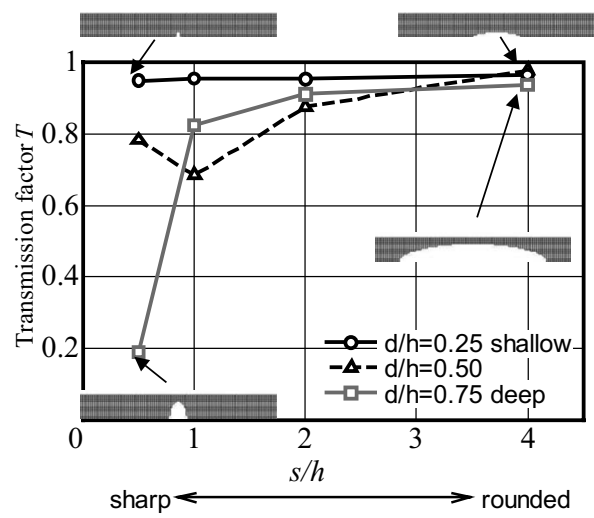

FIG. 6. Transmission factors for $\mathrm{A} 0$ incidence at a rounded defect of various shapes and depths. 


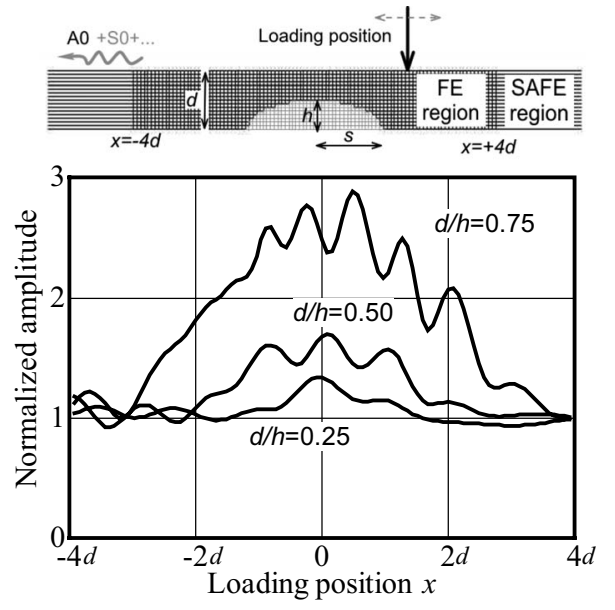

FIG. 7. Normalized amplitude for displacement in the thickness direction of an A0 mode for defects with various depths $(d / h=0.25,0.5,0.75)$ but the same shape $(s / h=4.0)$.

regions, the normal displacements of an A0 mode at the leftSAFE region are obtained, as shown in Fig. 7. Similar to Fig. 1 , the FE region is from $x=-4 d$ to $+4 d$, and a harmonic vibration of $f d=1.0 \mathrm{MHz} \mathrm{mm}$ is applied to the upper surface of the FE region. The center of the elliptical defect is at $x=0$, and the horizontal axis is in multiples of the thickness $d$. The normal displacement of A0 mode at the left region is normalized by the value when the normal loading is applied at the intact point $x=+4 d$. The amplitude distributions for defects of three different depths $(d / h=0.25,0.5,0.75)$ and fixed shapes $s / h=4.0$ are shown.

The amplitude distributions are roughly in inverse proportion to the depths $d / h$. However, the modulation in the defect region implies that there are some reflections and mode conversions, especially in a deep defect of $d / h=0.75$

The inverse amplitude of the distributions in Fig. 7 is graphed in Fig. 8 for the harmonic wave $f d=1.0 \mathrm{MHz} \mathrm{mm}$.

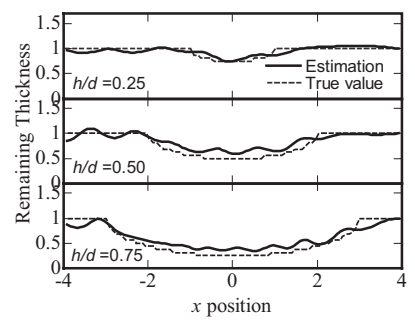

(a)

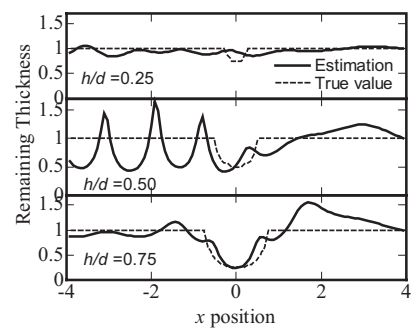

(c)

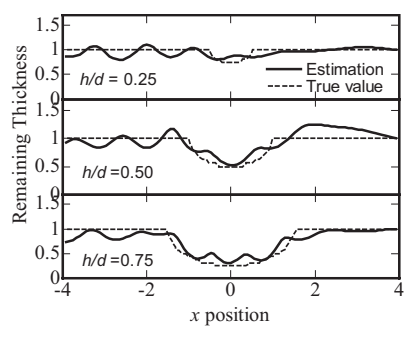

(b)

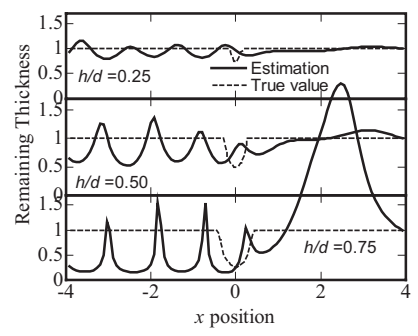

(d)
FIG. 8. Estimated remaining thickness for defects of various shapes and depths: (a) $s / h=4.0$, (b) $s / h=2.0$, (c) $s / h=1.0$, and (d) $s / h=0.5$.

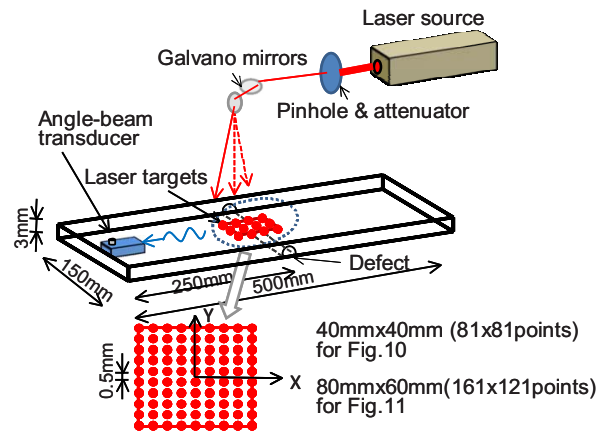

FIG. 9. (Color online) Sample and hardware setup.

The dashed lines denote the defect shape used in the calculation, while the solid lines are the estimated remaining thickness obtained from the amplitude distributions. For a rounded defect such as $s / h=4$ in Fig. 8(a), good agreement is found due to the small reflections and mode conversion. On the other hand, for sharper and deeper defects, which generate more reflected waves and larger mode conversions, the estimated values do not agree as well. For example, in Fig. 8(c) for $s / h=1.0$ and Fig. 8(d) for $s / h=0.5$, the peaks at the left of the defect are caused by the reflections from the defect, while the large values at the right are caused by the fact that the transmitted waves are small due to the sharp and deep defects.

\section{EXPERIMENTS}

In order to verify the remote thickness measurements using a SLS, experiments were carried out using aluminum plates of $3.0 \mathrm{~mm}$ thickness. Figure 9 shows the sample and experimental setup. A $Q$-switched $\mathrm{Nd} /$ yttrium aluminum garnet laser beam (Quantel Brilliant Ultra, 532-nm wavelength, $20-\mathrm{Hz}$ rate, 7.2-ns pulse duration, and 32-mJ pulse energy) was scanned rapidly in the $x$ and $y$ directions by two galvano mirrors placed about $300 \mathrm{~mm}$ away from the plate. The laser spot diameter was adjusted to about $1.0 \mathrm{~mm}$ by a pinhole to improve the spatial resolution of the images. The laser energy was attenuated to prevent damaging the surface of the specimens. An A0 Lamb-wave mode was detected by an angle-beam transducer composed of a $400-\mathrm{kHz} 1-3$ piezoelastic composite and a resin wedge. The detected radiofrequency signals were amplified by $56 \mathrm{~dB}$ by a preamplifier. Then, the signals were filtered by a fourth-order Butterworth low-pass filter of $400 \mathrm{kHz}$ and a high-pass filter of $600 \mathrm{kHz}$ to extract components below and above the A1 cutoff frequency of $f d=1.53 \mathrm{MHz} \mathrm{mm}$. Only direct signals were gated to prevent the influence of edge reflections. The signals were not averaged so that they could be measured as quickly as possible. The measurement spots on the aluminum plates were $81 \times 81$ points in $0.5-\mathrm{mm}$ steps over a range of $40 \times 40 \mathrm{~mm}^{2}$ in Fig. 10. In these cases, the distributions of estimated remaining thickness were obtained in about 10 min. Since the entire process of galvano scanner control, data acquisition, and display of results is now sequentially operated by LABVIEW software for the purpose of laboratory study, the measurements can be further speeded up by optimized acquisition done in ultrasonic microscopes. 

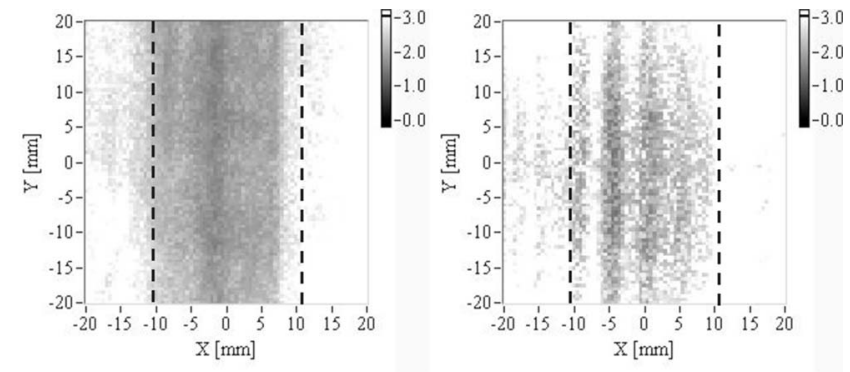

(a)

(b)
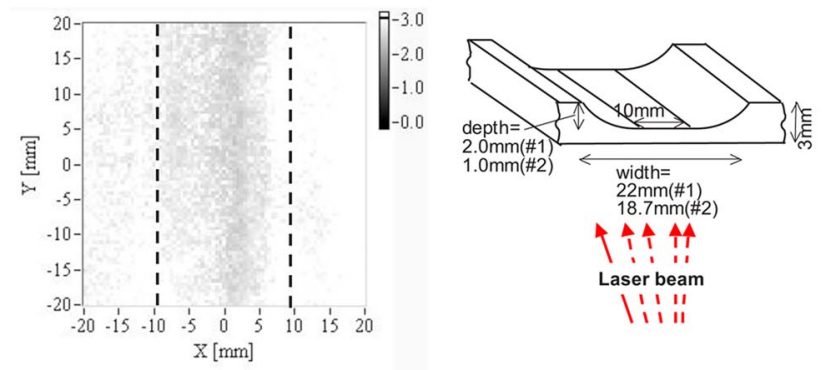

(c)

FIG. 10. (Color online) Estimated thickness distributions. (a) At defect 1, and a $400-\mathrm{kHz}$ LPF was applied to the signals. (b) At defect 1, a $600-\mathrm{kHz}$ HPF was applied to the signals. (c) At shallow defect 2, a 400-kHz LPF was applied to the signals.

The specimens used were aluminum plates measuring $150 \times 500 \times 3.0 \mathrm{~mm}^{3}$. Groove defects of $2.0-\mathrm{mm}$ (1) and $1.0-\mathrm{mm}$ (2) depths were machined at the center of the aluminum plates to match the calculation model. Both defects had a flat zone of $10-\mathrm{mm}$ width at the bottom, and the edges were cut by a ball end mill of $20-\mathrm{mm}$ diameter. The crosssection of these defects is shown in Fig. 10

Figure 10 shows the distribution of estimated remaining thickness obtained when a laser beam is incident on the flat surface of plates \#1 and \#2. In Fig. 10(a), the signals were filtered by a $400-\mathrm{kHz}$ low-pass filter to extract the frequency range below the A1 cutoff. The thickness distributions were obtained by inverting the normalized amplitude of the signals. In this case, the average value of the amplitudes detected at 81 points on the right edge of the figure is used for normalization. The dashed lines denote the edges of the dent, which agree with the area of the signal having large amplitude.

Figure 10(b) was obtained from the same experimental setup, but the signals were filtered by a $600-\mathrm{kHz}$ high-pass filter in order to show the result when unsuitable frequency range is used. In contrast to Fig. 10(a), several gray bands can be seen in the defect region due to large reflections and mode conversions in this frequency range.

In order to compare the distributions of remaining thickness for defects of different depths, Fig. 10(c) shows the distribution for a grooved defect of 1.0-mm depth (2). Similar to the case of a deep defect in Fig. 10(a), a laser beam was incident on the flat surface, and the signals were filtered by a $400-\mathrm{kHz}$ low-pass filter. The difference between the intact area of 3.0-mm thickness and the defect area is smaller than for the deep defect in Fig. 10, which represents that the defect in specimen 2 is shallower than that in specimen 1 .

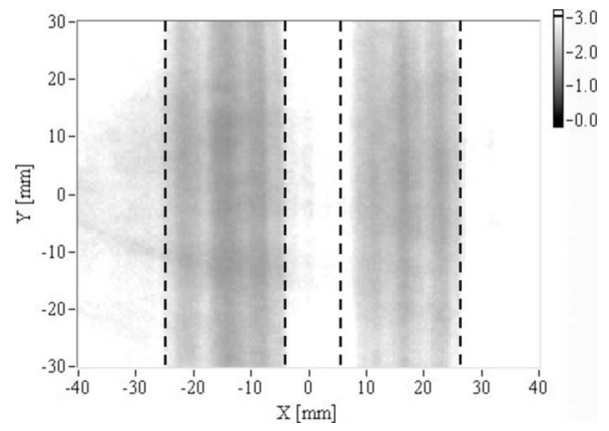

FIG. 11. Estimated thickness distribution for specimen 3 with two deep defects. The laser beams were incident on the flat surface, and a $400-\mathrm{kHz}$ LPF was applied to the signals.

To simulate more complex and realistic defects, specimen 3 has two grooved defects of 2.0-mm depth. The width and depth of defects are the same as 1 in Fig. 10(a), and the centerline of the two defects are $30 \mathrm{~mm}$ apart. Figure 11 shows the estimated remaining thickness obtained from the amplitude. Similar to Figs. 10(a) and 10(c) for 1 and 2, a laser beam was incident on the flat surface of the plate, and a $400-\mathrm{kHz}$ low-pass filter was applied to the signals. Two gray bands representing the grooved defects of 2.0-mm depth are clearly shown in Fig. 11, and the remaining thickness agrees roughly with the true value for both bands. This result demonstrates that remaining thickness can be evaluated even for the case of plural defects.

In these cases, remaining thickness in whole regions could be obtained with sufficient accuracy. However, directivity of an angle-beam transducer and attenuation and dispersion of an A0 mode may cause errors in an image of remaining thickness. In such cases, directivity of a receiving transducer can be improved by redesigning a transducer or by using multiple transducers, and variation in sensitivity due to attenuation and dispersion should be corrected from the propagation distance.

\section{CONCLUSIONS}

The present study described a rapid thickness measurement technique using the amplitude of the A0 mode of Lamb waves generated by laser emission. A combination calculation technique using a SAFE method and an ordinary FE method revealed that the $\mathrm{A} 0$ mode in the frequency range of $f d=1.0 \mathrm{MHz}$ mm below the A1 cutoff propagates through a shallow rounded defect with small reflections and mode conversions. The calculations also demonstrated that the A0 amplitude generated at a point source is roughly inversely proportional to the thickness at the source point. From such characteristics, the distributions of remaining thickness were experimentally obtained for aluminum plates with one and two grooved defects at high speed using a SLS and an anglebeam transducer.

Since laser-scanning using a galvano scanner is practical at high speeds, thickness measurements can be carried out much faster than in conventional contact-type thickness measurements by hand. Use of suitable equipment would provide a thickness image with the same speed as ultrasonic microscopes. 
An axisymmetric $L(0,1)$ mode propagates in pipes with similar characteristics as the A0 mode of a Lamb wave. Therefore, use of the $L(0,1)$ mode would enable this technique to be applied to thickness measurements of pipes.

${ }^{1}$ I. A. Victorov, Rayleigh and Lamb Waves (Plenum, New York, 1967).

${ }^{2}$ J. L. Rose, Ultrasonic Waves in Solid Media (Cambridge University Press, New York, 1999).

${ }^{3}$ P. Cawley, M. J. S. Lowe, D. N. Alleyne, B. Pavlakovic, and P. Wilcox, "Practical long range guided wave testing: Application to pipes and rails," Mater. Eval. 61, 66-74 (2003).

${ }^{4}$ H. Kwun, S. Y. Kim, and G. M. Light, "The magnetostrictive sensor technology for long range guided wave testing and monitoring of structures," Mater. Eval. 61, 80-84 (2003).

${ }^{5} \mathrm{~J}$. Li and J. L. Rose, "Excitation and propagation of nonaxisymmetric guided waves in a hollow cylinder," J. Acoust. Soc. Am. 109, 457-468 (2001).

${ }^{6}$ T. Hayashi, K. Kawashima, Z. Sun, and J. Rose, "Analysis of flexural mode focusing by a semi-analytical finite element method," J. Acoust. Soc. Am. 113, 1241-1248 (2003).

${ }^{7}$ T. Hayashi and M. Murase, "Defect imaging with guided waves in a pipe," J. Acoust. Soc. Am. 117, 2134-2149 (2005).

${ }^{8} \mathrm{~J}$. Davis and P. Cawley, "The application of synthetically focused imaging techniques for high resolution guided wave pipe inspection," in Review of Progress in Quantitative NDE, edited by D. Thompson and D. Chimenti (Plenum, New York, 2007), Vol. 26, pp. 681-688.

${ }^{9}$ D. N. Alleyne, M. J. S. Lowe, and P. Cawley, "The reflection of guided waves from circumferential notches in pipes," J. Appl. Mech. 65, 635-641 (1998).

${ }^{10}$ A. Demma, P. Cawley, M. Lowe, and A. G. Roosenbrand, "The reflection of the fundamental torsional mode from cracks and notches in pipes," J.
Acoust. Soc. Am. 114, 611-625 (2003).

${ }^{11}$ F. Benmeddour, S. Grondel, J. Assaad, and E. Moulin, "Study of the fundamental Lamb modes interaction with symmetrical notches," NDT \& E Int. 41, 1-9 (2008).

${ }^{12}$ P. A. Fomitchov, A. K. Kromin, S. Krishnaswamy, and J. D. Achenbach, "Imaging of damage in sandwich composite structures using a scanning laser source technique," Composites, Part B 35, 557-562 (2004).

${ }^{13}$ J. Takatsubo, B. Wang, H. Tsuda, and N. Tooyama, "Generation laser scanning method for the visualization of ultrasounds propagating on a 3-D object with an arbitrary shape," J. Solid Mechanics and Materials Eng. 1, 1405-1411 (2007).

${ }^{14}$ G. R. Liu and J. D. Achenbach, "Strip element method for stress analysis of anisotropic linearly elastic solids,” J. Appl. Mech. 61, 270-277 (1994).

${ }^{15}$ S. K. Datta, A. H. Shah, R. L. Bratton, and T. Chakraborty, "Wave propagation in laminated composite plates," J. Acoust. Soc. Am. 83, 2020-2026 (1988).

${ }^{16}$ T. Hayashi, W.-J. Song, and J. L. Rose, "Guided wave dispersion curves for a bar with an arbitrary cross-section, a rod and rail example," Ultrasonics 41, 175-183 (2003).

${ }^{17}$ T. Hayashi and J. L. Rose, "Guided wave simulation and visualization by a semi-analytical finite element method," Mater. Eval. 61, 75-79 (2003).

${ }^{18}$ Y. A. Al-Nassar, S. K. Datta, and H. Shah, "Scattering of Lamb waves by a normal rectangular strip weldment," Ultrasonics 29, 125-132 (1991).

${ }^{19}$ Y. Cho and J. L. Rose, "A boundary element solution for a mode conversion study on the edge reflection of Lamb waves," J. Acoust. Soc. Am. 99, 2097-2109 (1996).

${ }^{20}$ J. M. Galan and R. Abascal, "Numerical simulation of Lamb wave scattering in semi-infinite plates," Int. J. Numer. Methods Eng. 53, 1145-1173 (2002).

${ }^{21}$ T. Hayashi, "Guided wave animation using semi-analytical finite element method," in Proceedings of the 16th World Congress on Nondestructive Testing (2004), pp. 786-817. 